\title{
A Case of Refractory Oral Aphthous Ulcer Successfully Treated with Adalimumab
}

\author{
Jinyeong Kim ${ }^{1}$, Myung-jin Song ${ }^{1}$, Hye-jin Jeong ${ }^{2}$, Soo-Kyung Cho ${ }^{1,3}$, Yoon-Kyoung Sung ${ }^{1,3}$ \\ ${ }^{1}$ Department of Internal Medicine, Hanyang University College of Medicine, Seoul, ${ }^{2}$ Department of Rheumatology, Keimyung University \\ Dongsan Medical Center, Daegu, ${ }^{3}$ Department of Rheumatology, Hanyang University Hospital for Rheumatic Diseases, Seoul, Korea
}

\begin{abstract}
Oral aphthous ulcer is a common lesion characterized by loss of dermal tissue of various shapes in many diseases. It is not serious, but may affect quality of life through pain and discomfort. In many cases, it heals spontaneously without treatment or with topical agents such as antiseptics, analgesics and corticosteroid in a few days. However, rarely, there are a few cases of aphthous ulcer that remain refractory despite appropriate treatment. Tumor necrosis factor- $\alpha$ inhibitors have recently been used in treatment of severe mucosal ulcer in Behçet's disease. Herein, we report a patient suffering from refractory oral aphthous ulcers that were resistant to topical and systemic agents, but were successfully treated with adalimumab. (J Rheum Dis 2017;24:157-160)
\end{abstract}

Key Words. Oral ulcer, Aphthous stomatitis, Tumor necrosis factor-alpha inhibitor, Adalimumab

\section{INTRODUCTION}

Oral ulcer is a defect of surface tissue characterized by various depth, yellow-gray color fibrous membrane and relatively well-marked margin. It is associated with various conditions such as aphthous, trauma, drug, infection, malignancy and rheumatic disease including Behçet's disease. Among these, aphthous ulcer is the most common type which occurs in $20 \%$ of the general population at any time during their lifetime. Over than $80 \%$ of aphthous ulcers belong to minor type which tends to be smaller than $10 \mathrm{~mm}$ in diameter, self-limited and disappear not leaving a scar. Approximately $15 \%$ of aphthous ulcers are a major type which is larger than $10 \mathrm{~mm}$ in diameter, lasts from 2 weeks to several months and heals with scar [1]. In severe case of recurrent aphthous ulcers, particularly the major type, it is difficult to treat with topical agents alone. Therefore, systemic therapy with corticosteoid or immunesuppressive agent is necessary in recurrent aphthous ulcer [2]. Recently, it has been shown that tumor necrosis factor (TNF)- $\alpha$ inhibitors are used effectively for management of refractory and chroni- cally recurrent oral ulcers [3]. As anti-TNF- $\alpha$ therapy is largely effective for Behçet's disease characterized with multiple mucosal ulcers, we consider the use of TNF- $\alpha$ inhibitors for the patient without underlying disease, who is suffering from persistent oral ulcers. This case shows the successful management of refractory oral aphthous ulcers with adalimumab which is monoclonal antibody to TNF- $\alpha$.

\section{CASE REPORT}

A 62-year-old man, complaining persistent deep oral ulcers lasting for more than 2 years, was referred to our hospital. At the first time, oral ulcers were spontaneously developed on right border and tip of his tongue without any traumatic events. He visited dental clinic and was treated with local corticosteroid, anesthetics and antiseptics. Since there was no improvement in his symptoms, several times of local steroid injection and systemic corticosteroid was used besides topical agents. And additional supportive care was provided such as non-steroidal anti-inflammatory drug and analgesics for severe pain, pilo-

Received : June 15, 2016, Revised : August 30, 2016, Accepted : September 20, 2016

Corresponding to : Yoon-Kyoung Sung, Department of Rheumatology, Hanyang University Hospital for Rheumatic Diseases, 222 Wangsimni-ro, Seongdong-gu, Seoul 04763, Korea. E-mail : sungyk@hanyang.ac.kr 
carpine for dry mouth and antibiotic agent and anti-fungal agent for preventing secondary infection. Also, he was treated with surgical procedure that is excision with suture of mucous membrane three times, but it was failed due to wound dehiscence. Although various therapies were tried, oral ulcers were not improved and new lesions were developed at another site. Then he visited our hospital for challenging new treatments after 2 years from onset.

He had diabetes mellitus for over than 10 years with insulin therapy and it was not controlled strictly (hemoglobin A1c 7.7). He had not been diagnosed with hypertension, tuberculosis infection, chronic liver diseases, malignancy, sexually transmitted diseases and rheumatic diseases. There are no family history of severe oral-ulcer diseases and any rheumatic diseases.

A $20 \times 10 \mathrm{~mm}$ sized ulcer was located on left lower border of his tongue which had ovoid shape and clean-cut margin with soft tissue defect and whitish plaques were also accompanied around ulcer when he first visited. He had his weight loss about $5 \mathrm{~kg}$ for 2 years because of poor oral intake.

Initial laboratory findings were as follow: white blood cell count was $7,100 / \mathrm{mm}^{3}$ (absolute neutrophil count was $3,670 / \mathrm{mm}^{3}$ ); hemoglobin was $13.9 \mathrm{~g} / \mathrm{dL}$; platelet count was $248,000 / \mathrm{mm}^{3}$; erythrocyte sedimentation rate was $2 \mathrm{~mm} / \mathrm{hr}$; C-reactive protein was $<0.8 \mathrm{mg} / \mathrm{dL}$; serum creatinine was $0.72 \mathrm{mg} / \mathrm{dL}(0.5 \sim 1.2 \mathrm{mg} / \mathrm{dL})$; serum total protein was $6.8 \mathrm{~g} / \mathrm{dL}(6.4 \sim 8.5 \mathrm{~g} / \mathrm{dL})$; serum albumin was $4.2 \mathrm{~g} / \mathrm{dL}(3.2 \sim 5.5 \mathrm{~g} / \mathrm{dL})$; serum lactate dehydrogenase was $214 \mathrm{IU} / \mathrm{L}(60 \sim 200 \mathrm{U} / \mathrm{L})$.

Several systemic diseases which can cause characteristic oral ulcers should be ruled out. Clinical manifestations suggesting Behçet's disease including genital ulcers, eye lesions such as uveitis or retinal vasculitis, cutaneous lesions like erythema nodosum were not detected. Human leukocyte antigen-B51 was negative. Any cutaneous symptoms such as malar or discoid rash, arthritis and evidence of neurologic or hematologic diseases were not existed. Autoantibodies including anti-nuclear antibody, anti-SS-A/Ro antibody, anti-SS-B/La antibody and rheumatoid factor were all negative. He did not have systemic signs like febrile sensation or chilling. Human immunodeficiency virus and venereal disease research laboratory were negative. Simple chest X-ray appeared normal without old tuberculosis (TB) or other infectious sequelae. Sputum acid fast bacillus staining, purified protein derivative skin test and Interferon-gamma release assays of Mycobacterium tuberculosis were negative. Magnetic resonance imaging of his neck did not show the pathologic lymphadenopathy or malignant lesions. The Biopsy result of ulcer was benign which is ulcerative change with granulation tissue (chronic oral ulcer) rather than malignancy.

In our clinic, a medical treatment started with oral prednisolone up to $10 \mathrm{mg}$, colchicine $0.6 \mathrm{mg}$ twice a day and folic acid. After two months later, oral ulcer did not show any improvement and he felt still painful (Figure 1). We tried to apply azathioprine, but it was also not very helpful. Then, we discussed with patient to use TNF- $\alpha$ inhibitor
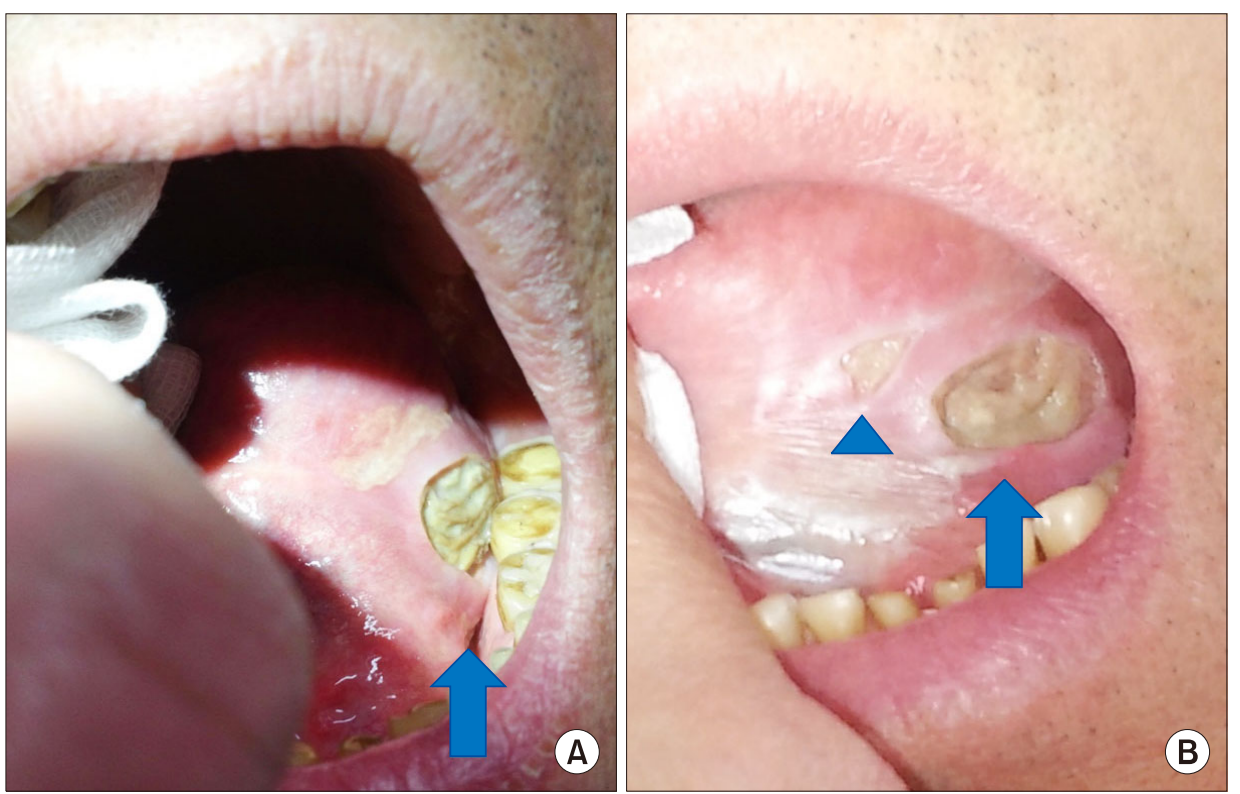

Figure 1. A $20 \times 10 \mathrm{~mm}$ sized oral ulcer (arrows) with deep clean-cut margin on his left border of tongue $(A)$, there was no any improvement but small extra-ulcer (arrow head) developed during systemic steroid therapy (B). 


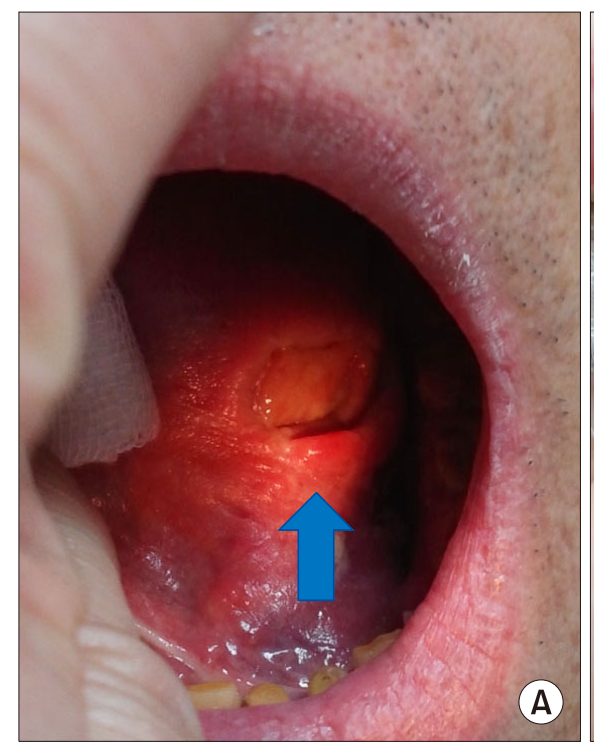

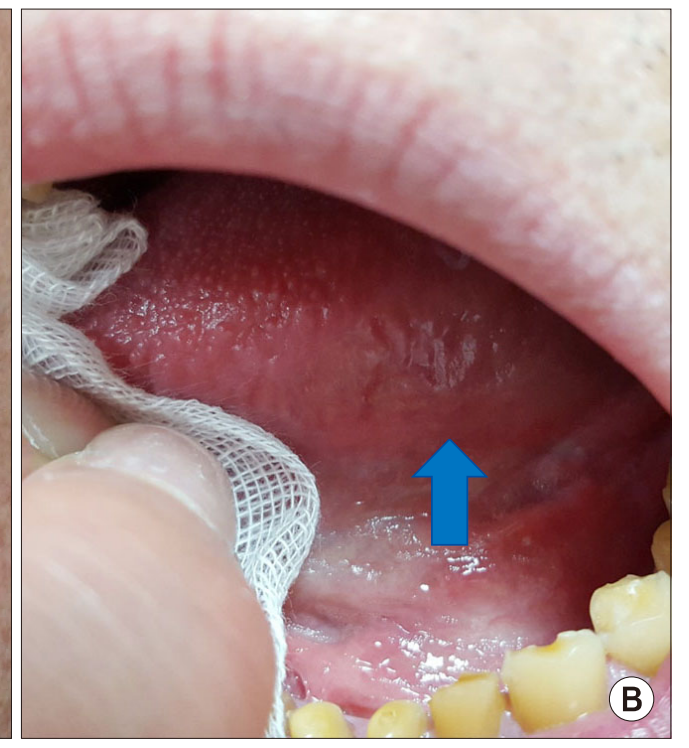

Figure 2. Healing ulcer (arrows) at 2 months (A), and 7 months (B) from administration of adalimumab. and started subcutaneous injection of adalimumab $40 \mathrm{mg}$ every 2 weeks. The dose of oral corticosteroid got tapered steadily out after 5 th injection and completely stopped after 11th injection. Interval of administration of adalimumab also extended from every 2 to 4 weeks. After two months later starting treatment with adalimumab, the patient had an improvement of pain. And oral ulcer was completely healed within seven months of treatment (Figure 2). He has remained in remission state and followed up in the outpatient department maintaining adalimumab 40 mg every 4 weeks without systemic corticosteroid.

\section{DISCUSSION}

Oral ulcers are classified into two groups which are acute and chronic according to their duration. Acute ulcers develop within short time and last less than 2 weeks. On the other hand, chronic ulcers slowly develop and progress consistently. Common causes of oral ulcer are trauma including mechanical and chemical, drug-induced, autoimmune disorders, infectious diseases such as tuberculosis, mycosis, other bacterial and parasite, and malignancy [4]. Our patient had 2-years lasting oral ulcer which was not easily cured with appropriate therapy. We tried to find his underlying disease which can induce intractable and persisting ulcer. We should have focused on aphthous ulcer itself because we could not find specific systemic disease to him for evaluation.

Generally, treatment of oral aphthous ulcer starts with topical agents such as local anesthetics, antiseptic/anti-inflammatory agents, sucralfate and local corticosteroid. If painful ulcers prolong due to failure of topical medications, a systemic therapy is indicated which includes colchicine, corticosteroid, pentoxifylline, dapsone, anti-metabolites, cyclosporine and alkylating agents. He was treated with from topical agents to systemic corticosteroid and colchicine. All these medical treatment and even surgical repair were failed. Moreover, we could not increase the usage of corticosteroid because of his diabetes mellitus. Because it is generally accepted that higher occurrence of oral mucosal lesions was significantly associated with poor metabolic control of diabetes mellitus [5]. Azathioprine also was added to decrease dose of corticosteroid, but it did not affect at all. So, It was proven that his oral aphthous ulcer was refractory to conventional treatments.

TNF- $\alpha$ is an immune-modulating cytokine as important mediator in various aspects of immunity. The unregulated TNF- $\alpha$ expression develops immune diseases. One study reported that oral ulcer is characterized by high expression of TNF- $\alpha$. This major inflammatory mediator may contribute to the activation and recruitment of leukocytes that are found in oral ulcers [6]. There are currently 5 TNF- $\alpha$ inhibitors approved in rheumatic diseases which are divided two strategies: soluble receptors (etanercept) and targeting monoclonal antibodies (infliximab, adalimumab, golimumab, certolizumab). A recent report has shown that the effectiveness of adalimumab therapy in autoimmune disease had better outcomes than that of infliximab and etanercept from a molecular view [6]. The more effective antigen-antibody interface in adalimumab provided information at a molecular structural 
level for further understanding the clinical advantages of adalimumab therapy compared with infliximab [7]. Based on such studies, we chose adalimumab among TNF- $\alpha$ inhibitors in spite of the cost from its off-label use.

Like all biologics, anti TNF- $\alpha$ agents should be used with caution because of their side effects. The common side effects are injection site reaction and upper respiratory infections. They do not necessitate the termination of injection but need supportive supervision by specialists. More serious side effects include tuberculosis reactivation, deep fungal injections and infection from other atypical pathogens. Rarely, worsening or initiation of congestive heart failure, medically significant cytopenia, development of lymphoma can be possibly notable [8]. Patient was observed closely by medical expert during first administration of adalimumab. To prevent possible infection, we screened his latent tuberculosis infection and vaccinated him from pneumococcus infection. Also serial laboratory monitoring for liver enzymes was done. Besides medical problems, high cost from off-label use might be bother patient absolutely.

One study shows the characteristics of patient-group of recurrent aphthous ulcer [9]. It causes subjective pain in $90 \%$ of patients, as well as emotional stress. It can induce difficulty to eat and swallow in $80 \%$ of them, causing malnutrition and poor quality of their lives. Considering his occupation-professor, it might become his handicap causing a pronunciation problem. Although it is not being life-threatening diseases, these ulcers must be treated aggressively. With intractable patient with oral ulcer, TNF- $\alpha$ inhibitors are brought up and those are able to prevent unnecessary use of systemic corticosteroid.

There has been two similar cases that intractable oral ulcers without underlying diseases such as Behçet's or Crohn's disease were treated with adalimumab $[10,11]$. In all cases, ulceration seemed to be resistant to conventional therapies, prior to use adalimumab. The difference of our case from those reports is the route of administration of adalimumab-subcutaneously injected in reference cases.

This study is the first to report the remission of intractable aphthous oral ulcer without underlying disease by administrating adalimumab in Korea. There has been a limitation to assess an effect of adalimumab in oral aphthous ulcer from a lack of cases or studies. Therefore, further studies based on large number of patients for longer duration in order to confirm long-term safety and effectiveness in oral aphthous ulcers are necessary.

\section{SUMMARY}

Here we report the case of successful treatment of a 62-year old man with intractable oral aphthous ulcer with adalimumab. This suggests that application of anti-TNF- $\alpha$ therapy can be effective for the management of recalcitrant oral ulcer.

\section{CONFLICT OF INTEREST}

No potential conflict of interest relevant to this article was reported.

\section{REFERENCES}

1. Field EA, Allan RB. Review article: oral ulceration-- aetiopathogenesis, clinical diagnosis and management in the gastrointestinal clinic. Aliment Pharmacol Ther 2003;18:94962.

2. Beguerie JR, Sabas M. Recurrent aphthous stomatitis: an update on etiopathogenia and treatment. J Dermatol Nurses Assoc 2015;7:8-12.

3. Altenburg A, Abdel-Naser MB, Seeber H, Abdallah M, Zouboulis CC. Practical aspects of management of recurrent aphthous stomatitis. J Eur Acad Dermatol Venereol 2007;21:1019-26.

4. Muñoz-Corcuera M, Esparza-Gómez G, González-Moles MA, Bascones-Martínez A. Oral ulcers: clinical aspects. A tool for dermatologists. Part II. Chronic ulcers. Clin Exp Dermatol 2009;34:456-61.

5. Al-Maweri $\mathrm{SA}$, Ismail $\mathrm{NM}$, Ismail $\mathrm{AR}, \mathrm{Al}-G h a s h m ~ \mathrm{~A}$. Prevalence of oral mucosal lesions in patients with type 2 diabetes attending hospital universiti sains malaysia. Malays J Med Sci 2013;20:39-46.

6. Natah SS, Häyrinen-Immonen R, Hietanen J, Malmström $\mathrm{M}$, Konttinen YT. Immunolocalization of tumor necrosis factor-alpha expressing cells in recurrent aphthous ulcer lesions (RAU). J Oral Pathol Med 2000;29:19-25.

7. Hu S, Liang S, Guo H, Zhang D, Li H, Wang X, et al. Comparison of the inhibition mechanisms of adalimumab and infliximab in treating tumor necrosis factor $\alpha$-associated diseases from a molecular view. J Biol Chem 2013; 288:27059-67.

8. Scheinfeld N. Adalimumab: a review of side effects. Expert Opin Drug Saf 2005;4:637-41.

9. Safadi RA. Prevalence of recurrent aphthous ulceration in Jordanian dental patients. BMC Oral Health 2009;9:31.

10. Vujevich J, Zirwas M. Treatment of severe, recalcitrant, major aphthous stomatitis with adalimumab. Cutis 2005;76: 129-32.

11. Sánchez-Cano D, Callejas-Rubio JL, Ruiz-Villaverde R, Ortego-Centeno N. Recalcitrant, recurrent aphthous stomatitis successfully treated with adalimumab. J Eur Acad Dermatol Venereol 2009;23:206. 